\title{
Lack of preclinical support for the efficacy of histone deacetylase inhibitors in the treatment of psoriasis.
}

Anna-Karin Ekman and Charlotta Enerbäck

\author{
Linköping University Post Print
}

\section{Tweet}

N.B.: When citing this work, cite the original article.

Original Publication:

Anna-Karin Ekman and Charlotta Enerbäck, Lack of preclinical support for the efficacy of histone deacetylase inhibitors in the treatment of psoriasis, 2016, British Journal of Dermatology, (174), 2, 424-426.

http://dx.doi.org/10.1111/bjd.14021

Copyright: Wiley: 12 months

http://eu.wiley.com/WileyCDA/

Postprint available at: Linköping University Electronic Press

http://urn.kb.se/resolve?urn=urn:nbn:se:liu:diva-125471 


\section{Lack of preclinical support for the efficacy of HDAC inhibitors in the}

\section{treatment of psoriasis}

AK Ekman and C Enerbäck

Ingrid Asp Psoriasis Research Center, Department of Clinical and Experimental Medicine, Faculty of Health Sciences, Linköping University, Linköping, Sweden

Running title: Lack of support for HDAC inhibitors in psoriasis

Funding sources: The study was funded by the Ingrid Asp Foundation, the Welander Foundation and the Swedish Psoriasis Association.

Conflict of interest: The authors have no conflict of interest.

Word count: 1000

\section{Corresponding author:}

Charlotta Enerbäck, Ingrid Asp Psoriasis Research Center, Department of Clinical and Experimental Medicine, Linköping University, SE-581 85 Linköping, Sweden

Phone +46101037429 E-mail: charlotta.enerback@liu.se 


\section{To the editor}

Histone acetylation and deacetylation is an important epigenetic regulator of gene expression. The acetylation of histones leads to a more accessible chromatin state and enables gene transcription. Histone deacetylases (HDACs) alter gene expression by removing the acetylation of the histones, resulting in a more condensed chromatin state, which in turn leads to repressed gene transcription. Consequently, the inhibition of histone deacetylation promotes gene expression. The acetylation of histones is believed to be primarily regulated by the HDAC-1, -2 and -3 proteins ${ }^{1}$, which belong to the class I HDACs.

Inhibitors of HDACs are emerging as promising novel agents for the treatment of a range of disorders, including cancer, neurodegenerative conditions and inflammatory diseases ${ }^{2,3}$. An abnormal expression of HDACs may alter the inflammatory response. In rheumatoid arthritis (RA), which shares both genetic susceptibility genes and immunopathogenesis with psoriasis, an increased expression and activity of HDAC-1 has been demonstrated in both PBMCs ${ }^{4}$ and fibroblast-like synoviocytes ${ }^{5}$. Moreover, HDAC inhibitors have shown clinical efficacy both in animal models of RA and in other inflammatory conditions, as well as in individuals with juvenile arthritis ${ }^{5}$.

Several in vitro studies of HDAC inhibitors have revealed an anti-inflammatory, antiproliferative and anti-angiogenic effect, suggesting that they may be effective in targeting the three main features of psoriasis pathogenesis: inflammation, excessive keratinocyte proliferation and angiogenesis. Treatment with HDAC inhibitors decreases the LPS-induced expression levels of tumor necrosis factor (TNF)- $\alpha$, interleukin (IL)- $1 \beta$ and IL- $6{ }^{6}$, prevents dendritic cell differentiation and promotes the generation of regulatory $\mathrm{T}$ cells ${ }^{7}$. The inhibition of HDACs induces growth arrest in several cell types, including keratinocytes ${ }^{8}$. Finally, HDAC inhibitor treatment reduces endothelial cell proliferation, migration and tube formation, supporting the hypothesis that the inhibitors have anti-angiogenic properties ${ }^{9}$. 
The effect of HDAC inhibitors in psoriasis has not yet been described, but the benefit of these compounds in other inflammatory conditions and in the modulation of inflammation, proliferation and angiogenesis after in vitro treatment makes it tempting to speculate on a possible role in the treatment of psoriasis ${ }^{10}$.

To investigate whether HDACs are dysregulated in psoriasis, we assayed the total HDAC activity in peripheral blood mononuclear cells (PBMCs) and skin from psoriasis patients and characterized the protein expression of HDAC-1, -2 and -3 in the psoriatic skin. PBMCs were isolated from 15 psoriasis patients and 15 healthy controls. The PBMCs were cryopreserved in liquid nitrogen until the time of the assay, when they were suspended in hypotonic buffer (10 mM EDTA, $50 \mathrm{mM}$ Tris) and lysed to obtain the protein fraction.

Skin punch biopsies were obtained from 5 psoriasis patients and 5 healthy controls and were immediately stabilized in Allprotect ${ }^{\mathrm{TM}}$ reagent (QIAGEN, Hilden, Germany). Total protein was extracted by snap-freezing in liquid nitrogen, followed by mechanical disruption and lysis buffer (hypotonic buffer with 1\% Triton-X). The obtained suspension was snap-frozen and stored overnight.

A total of $50 \mu$ g protein was used from each sample in an HDAC activity assay (BioVision, Milpitas, CA, USA), according to the instructions of the manufacturer. The activity assay was performed on total protein and measures the activity of all HDACs with the capacity to deacetylate histones. The resulting color intensity was measured at $405 \mathrm{~nm}$ and the standard supplied in the assay was used to prepare a reference curve of HDAC activity. Statistical analysis of the activity assay was performed using the Mann-Whitney test for non-parametric, unpaired samples. A p-value of less than 0.05 was considered significant.

For immunohistochemical detection of the expression of HDAC-1, HDAC-2 and HDAC-3 protein, punch biopsies were obtained from the lesional skin of 4 psoriasis patients and from the normal skin of 4 healthy controls. The biopsies were fixed in formaldehyde, dehydrated 
and embedded in paraffin. The paraffin-embedded biopsies were sectioned and stained with rabbit polyclonal anti-HDAC-1 (dilution 1/2; Abcam, Cambridge, UK), mouse monoclonal anti-HDAC-2, clone HDAC2-62 (dilution 1/2,000; Abcam) or rabbit polyclonal anti-HDAC3 (dilution 1/100; Abcam), using a standard immunohistochemistry protocol and the DAKO EnVision system (DAKO, Glostrup, Denmark). Negative controls were obtained by omitting the primary antibody.

Although all samples displayed signs of HDAC activity, we observed no differences in the activity of PBMCs from psoriasis patients compared with healthy controls (Fig. 1A). Nor did we see any difference in the activity of protein extracted from lesional psoriasis biopsies compared with healthy skin (Fig. 1B). While the sample size is small, a prominent ant clinically relevant change in activity should be visible even in a small sample size. The diversity in response even in healthy control subjects suggests that there is a large variation in HDAC activity between individuals even when psoriasis is not a factor and that other parameters influence the activity to a greater extent than having psoriasis.

Immunohistochemical staining revealed the presence of HDAC-1 and -2 protein in both lesional psoriatic skin and healthy skin, localized primarily to the epidermis. For HDAC-3, the staining varied between individuals, but these variations were found among both healthy controls and psoriasis patients. None of the antibody stainings revealed any difference in distribution or intensity between healthy controls and psoriasis patients.

A dysregulation of HDACs has been described in a variety of diseases, including RA ${ }^{4}$, ankylosing spondylitis ${ }^{11}$, different cancer types ${ }^{12}$ and cardiovascular disease ${ }^{13}$. The use of HDAC inhibitors as pharmacological treatment is an expanding field with promising results for the treatment of several of these diseases ${ }^{14}$. In lesional psoriatic skin, an over-expression 
of HDAC-1 mRNA has been previously suggested ${ }^{15}$, but has not been confirmed at protein level. Nor has the HDAC activity in psoriasis been previously determined.

In this study we have chosen to focus on the class I HDACs because of their prominent role in histone deacetylation. We cannot draw any conclusions about whether other epigenetic mechanisms, for example methylation, have a role in psoriasis pathogenesis.

In this study, we examined the levels of total HDAC activity in blood and skin in psoriasis and investigated the protein expression of HDAC-1, HDAC-2 and HDAC-3 in the psoriatic lesion. We obtained no preclinical support for a dysregulation of HDACs in psoriasis.

This lack of difference in HDAC activity and HDAC-1, -2 and -3 protein expression between psoriatic lesions and healthy skin further suggests that HDAC inhibitors may not be a relevant treatment for this patient group. 


\section{Acknowledgements}

The study was funded by the Ingrid Asp Foundation, the Welander Foundation and the Swedish Psoriasis Association. 


\section{Figure legends}

Figure 1. HDAC activity in (A) peripheral blood mononuclear cells (PBMCs), $n=15$, isolated from psoriasis patients or healthy controls, and (B) skin biopsies, $n=5$, from psoriatic lesions or from healthy controls. The line displays the mean value.

Figure 2. Protein expression of HDAC-1 (A, B), HDAC-2 (C, D) and HDAC-3 (E, F) in healthy skin (A, C, E) and lesional psoriatic skin (B, D, F) visualized using brown 3,3’diaminobensidine (DAB) immune staining. Sections are counterstained with hematoxylin. No DAB staining was observed when primary antibodies were omitted (negative control, $\mathbf{G}$ for anti-mouse and $\mathbf{H}$ for anti-rabbit). Immunohistochemistry was performed on lesional psoriatic skin from four patients and healthy skin from four healthy controls. The pre-diluted antiHDAC-1 antibody was applied at a further dilution of 1/2, the anti-HDAC-2 antibody was applied at a dilution of 1/2,000 and the anti-HDAC-3 antibody was used in a 1/100 dilution. 


\section{References}

1 Newbold A, Matthews GM, Bots M et al. Molecular and biologic analysis of histone deacetylase inhibitors with diverse specificities. Mol Cancer Ther 2013; 12: 2709-21.

2 Li J, Li G, Xu W. Histone deacetylase inhibitors: an attractive strategy for cancer therapy. Curr Med Chem 2013; 20: 1858-86.

3 Shanmugam MK, Sethi G. Role of epigenetics in inflammation-associated diseases. Subcell Biochem 2013; 61: 627-57.

4 Gillespie J, Savic S, Wong C et al. Histone deacetylases are dysregulated in rheumatoid arthritis and a novel histone deacetylase 3-selective inhibitor reduces interleukin-6 production by peripheral blood mononuclear cells from rheumatoid arthritis patients. Arthritis Rheum 2012; 64: 418-22.

5 Hawtree S, Muthana M, Wilson AG. The role of histone deacetylases in rheumatoid arthritis fibroblast-like synoviocytes. Biochemical Society transactions 2013; 41: 783-8.

6 Leoni F, Zaliani A, Bertolini G et al. The antitumor histone deacetylase inhibitor suberoylanilide hydroxamic acid exhibits antiinflammatory properties via suppression of cytokines. Proc Natl Acad Sci U S A 2002; 99: 2995-3000.

7 Falkenberg KJ, Johnstone RW. Histone deacetylases and their inhibitors in cancer, neurological diseases and immune disorders. Nature reviews. Drug discovery 2014; 13: 67391.

8 Saunders N, Dicker A, Popa C et al. Histone deacetylase inhibitors as potential anti-skin cancer agents. Cancer Res 1999; 59: 399-404.

9 Kwon HJ, Kim MS, Kim MJ et al. Histone deacetylase inhibitor FK228 inhibits tumor angiogenesis. Int J Cancer 2002; 97: 290-6.

10 McLaughlin F, La Thangue NB. Histone deacetylase inhibitors in psoriasis therapy. Curr Drug Targets Inflamm Allergy 2004; 3: 213-9.

11 Toussirot E, Abbas W, Khan KA et al. Imbalance between HAT and HDAC activities in the PBMCs of patients with ankylosing spondylitis or rheumatoid arthritis and influence of HDAC inhibitors on TNF alpha production. PLoS One 2013; 8: e70939.

12 Glozak MA, Seto E. Histone deacetylases and cancer. Oncogene 2007; 26: 5420-32.

13 Wang Y, Miao X, Liu Y et al. Dysregulation of histone acetyltransferases and deacetylases in cardiovascular diseases. Oxid Med Cell Longev 2014; 2014: 641979.

14 Tang J, Yan H, Zhuang S. Histone deacetylases as targets for treatment of multiple diseases. Clin Sci (Lond) 2013; 124: 651-62.

15 Tovar-Castillo LE, Cancino-Diaz JC, Garcia-Vazquez F et al. Under-expression of VHL and over-expression of HDAC-1, HIF-1alpha, LL-37, and IAP-2 in affected skin biopsies of patients with psoriasis. Int J Dermatol 2007; 46: 239-46. 

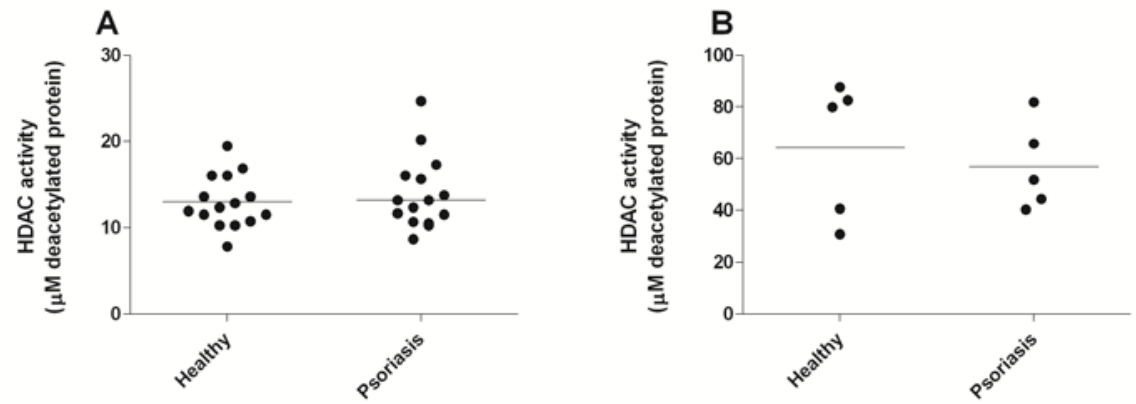

Figure 1. 

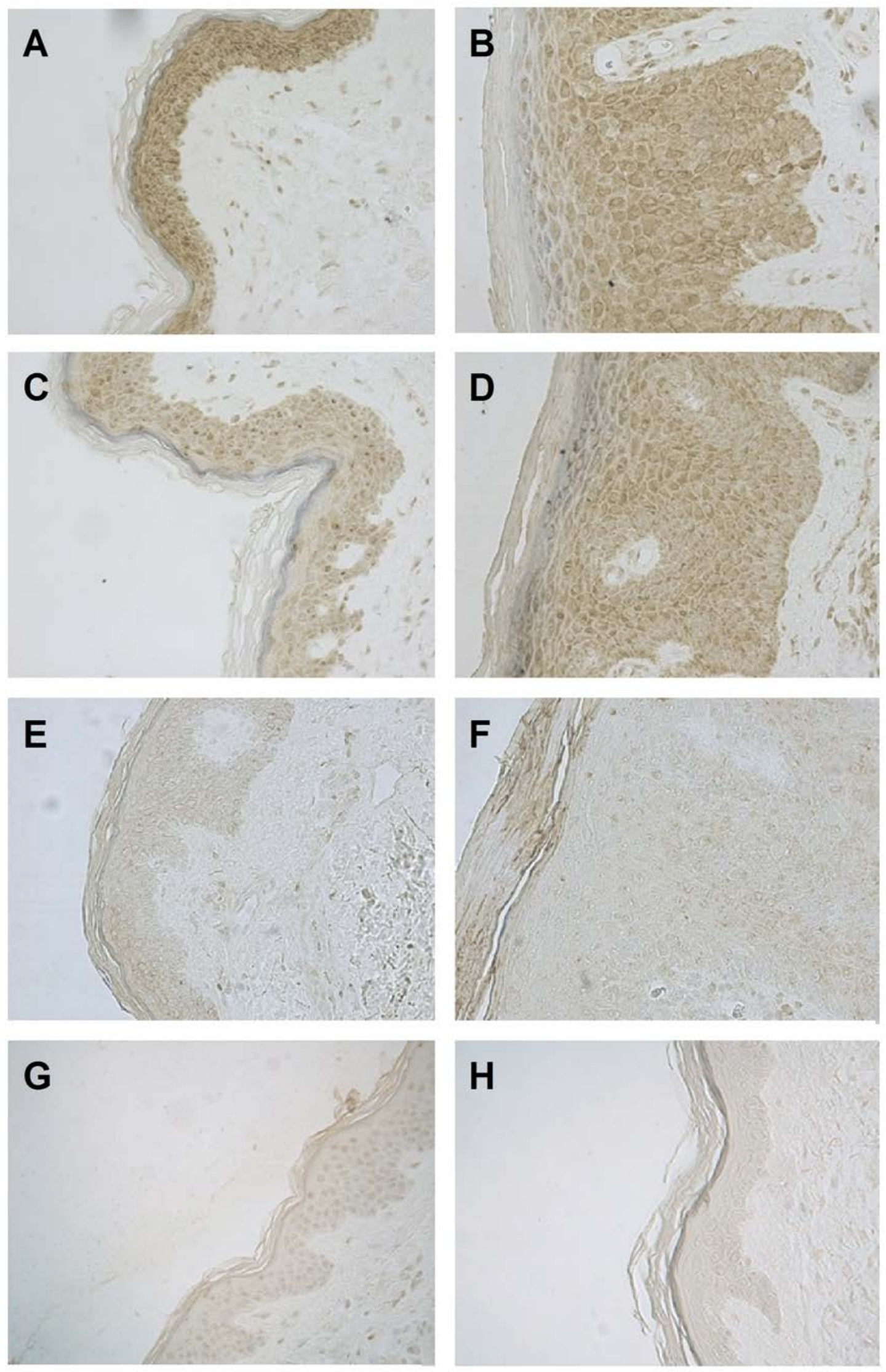

Figure 2. 